\title{
Interpreting two-photon imaging data of lymphocyte motility
}

\author{
Michael E. Meyer-Hermann ${ }^{1,2, *}$ and Philip K. Maini ${ }^{1}$ \\ ${ }^{1}$ Centre for Mathematical Biology, Mathematical Institute, Oxford University, 24-29 St. Giles', Oxford OX1 3LB, \\ United Kingdom \\ ${ }^{2}$ Frankfurt Institute for Advanced Studies (FIAS), Johann Wolfgang Goethe-University, Max von Laue Str. 1, \\ 60438 Frankfurt/Main, Germany
}

(Received 17 November 2004; published 22 June 2005)

\begin{abstract}
Recently, using two-photon imaging it has been found that the movement of $B$ and $T$ cells in lymph nodes can be described by a random walk with persistence of orientation in the range of 2 minutes. We interpret this new class of lymphocyte motility data within a theoretical model. The model considers cell movement to be composed of the movement of subunits of the cell membrane. In this way movement and deformation of the cell are correlated to each other. We find that, indeed, the lymphocyte movement in lymph nodes can best be described as a random walk with persistence of orientation. The assumption of motility induced cell elongation is consistent with the data. Within the framework of our model the two-photon data suggest that $T$ and $B$ cells are in a single velocity state with large stochastic width. The alternative of three different velocity states with frequent changes of their state and small stochastic width is less likely. Two velocity states can be excluded.

DOI: 10.1103/PhysRevE.71.061912

PACS number(s): 87.17.- d, 87.16.-b, 87.64.Vv
\end{abstract}

\section{INTRODUCTION}

The method of two-photon imaging has opened up a new way to generate data on cell motility in vivo. This method allows tracking of cell movement in living organs with only minimum disturbance. Therefore, the behavior of the cells can be considered to be representative of real in vivo behavior. Recently, this method has been applied to $B$ cells (BC) and $T$ cells (TC) in lymph nodes of mice [1,2]. Lymph nodes are prominent secondary lymphoid organs in which the interaction of lymphocytes and antigen presenting cells is organised. The motility of lymphocytes within secondary lymphoid organs is still a matter of controversy. BC and TC express and regulate chemokine receptors [3-5] and may therefore move according to chemotaxis or haptotaxis. In contrast to this hypothesis, two-photon data seem to favor a random walk of lymphocytes at least in the outer region of lymph nodes including primary follicles [6].

We aim to develop a model that describes lymphocyte migration in secondary lymphoid organs and revisit the interpretation of the results found with two-photon imaging. As the data show a stochastic variation of cell motility we are restricted to the class of stochastic models. Assuming that cell shape and motility are closely interlinked with each other [7], the cell cannot be represented by a point but has to be spatially resolved to some extent. Thus, within lattice descriptions, the lattice has to be of higher resolution than the cell diameter. Alternatively, one may introduce flexible cell objects as was done in [8-10], and which will be discussed separately.

The extended Potts model is the classical approach for the description of cells at a subcellular level, i.e., including prop-

\footnotetext{
*Author to whom correspondence should be addressed: Frankfurt Institute for Advanced Studies (FIAS), Johann Wolfgang GoetheUniversity, Max von Laue Str. 1, 60438 Frankfurt/Main, Germany. Email address: M.Meyer-Hermann@fias.uni-frankfurt.de
}

erties such as cell shape, surface molecules, organelles, or other internal structures [11]. It is derived from the Ising model and extends the latter to multiple spin states. A cell is defined by all volume elements that are in the same spin state. Movements, or more general changes on the lattice, are driven by a Boltzmann law at some biological temperature. The contributions to the energy term in the Boltzmannexponential define the cellular dynamics and interactions. In slightly more general terms the Potts model can be described as a potential-based thermodynamical model.

A cell subunit has to adopt the spin states of its neighbor point for cell movement. In this way every cell movement is intrinsically correlated with a change of cell volume. On longer time scales these volume fluctuations are averaged out, at least if a volume conserving potential is included in the energy entering the Boltzmann law. A peculiarity of this concept is that cells composed of one volume element only are in some sense immobile: Either the cell with a single volume element disappears by a spin flip or it doubles its volume. It is inferred that if the Potts model is to be applied to cells with small numbers of subunits, additional rules have to be included.

In the following we discuss an alternative model architecture which intrinsically includes the one- and the multisubunit limit within one concept. This opens up the possibility of determining the lattice constant by the size of the smallest cell in the system under consideration, which then would be described by a single volume element. The CPU load could be limited in this way when it is not the substructure of the cells that is interesting but their differences in volume.

As cells are widely incompressible objects we aim to use a mechanism of cell movement that conserves the total volume of the cell (if no growth or shrinking is intended). In our new model, denoted by the Greek word for tissue hyphasma, in the following, all reaction kinetics are formulated as reaction rates and actions are taken according to probabilistic decisions. In contrast to the potential-based Potts model the 
physical movement of the cells is formulated in terms of forces acting on subunits of the cell.

In this paper we will first introduce the model concept in Sec. II and then apply it to lymphocytes. We will analyze and interpret two-photon motility data in the framework of $h y$ phasma in Sec. III.

\section{DEFINITION OF THE MODEL}

\section{A. General considerations}

The new model for cell motility aims at capturing cell deformations and displacement in an agent-based model concept. Single subunit agent-based models are excluded for three reasons: First, the cell volume is not adequately represented. Second, cell deformations can not be explicitly represented. Finally, the velocity distributions as found in the two-photon imaging experiments cannot be reproduced. The last point follows from the fact that displacements have been recorded at intervals of 10 minutes: The lattice resolution is determined by the cell volume for single subunit models. At that resolution the cells have to overcome a large distance within a single time step for every movement. In order to respect the average velocity such large distance movements have to be rare events, thus leading to a rather discontinuous velocity distribution (in contrast to the experimental results). Thus a correct description of cell volume and velocity distribution exclude each other in a single subunit agent-based model.

In the two-photon imaging experiment cell tracks were projected onto a two-dimensional plane. The authors found that most cells "preferentially moved parallel to the overlying capsule" [1]. Therefore, as a first step, two-dimensional simulations can be considered as a good approximation for these data. The influence of the third dimension will be analyzed in future work.

The model hyphasma is based on rather simple assumptions, thus following a reductionalist point of view. Cell objects are represented by the cell volume, the cell polarity $\vec{p}$ (defined in Sec. II B), a list of cell subunits, and internal velocity states. The cell volume determines the number of cell subunits $N$ according to the space resolution $\Delta x$. The velocity state $\bar{v}$ is translated into probabilities of subunit movements $p_{\text {move }}$ in the direction of the cell polarity $\vec{p}$ according to the time resolution $\Delta t$ (compare Sec. II B).

Cell velocity states are observable in experiments but they are difficult to relate to internal cell properties. The velocities $\vec{v}_{i}$ of the subunits as observed in the model results, however, may be related to physical forces $\vec{F}_{i}$ according to the overdamped force equation:

$$
\vec{v}_{i}=\vec{F}_{i} / \gamma_{i},
$$

where $\gamma_{i}$ represents friction and $i$ denotes the subunit under consideration. In this picture the velocities of the subunits are the result of a force balance (that may include reshaping cell forces, forces due to active cell motility towards a chemokine, etc.). The $\gamma_{i}$ arise as a result of a number of processes, for example, the viscosity of the cytosol within a cell, and adhesion between cells. Note, however, that a force balance equation for the cell subunits is not necessarily a correct description of active processes of deformation and reshaping of cells. Thus the interpretation of cell subunit velocities in terms of forces has to be considered as an approximation to more complex internal processes within the cell. In the present investigation we do not make use of this interpretation and focus on the movement of free cells, thus neglecting chemotaxis and adhesion to other cells. Thus we directly use the cell velocity as found in experiment as input for the model.

In the following we base the kinetics of the cell subunits on two types of velocity: (i) undirected active movement with persistence of orientation, and (ii) cell reshaping.

\section{B. Active cell movement}

The normed orientation vector $\vec{p}$ determines the direction of the active movement of a cell. This vector represents an overall polarity of the cell which in reality is a complex function of internal organisation of the cytoskeleton as well as localized signal pathways. $\vec{p}$ may be considered as an approximation for the direction in which protrusions are developed by the cell that induce cell movement. The orientation vector is assumed to change randomly with a probability per time step that represents the persistence time $\Delta t_{\text {persist }}$, i.e., the inverse rate of change of orientation.

Each individual cell has at least one active velocity state $\bar{v}_{\text {active }}$ determining the probability of subunit movement in direction $\vec{p}$. The active movement of the cell is performed by the following procedure (see Fig. 1): The barycenter of the cell $\vec{b}$ is virtually shifted in the direction of $\vec{p}$ to the border of the cell $\vec{b}_{\text {virtual }}$. Then every subunit representing a border point of the cell is moved in random order towards free lattice points near the virtual barycenter [see Eq. (2)]. When a border subunit which is not a direct neighbor of its target point (e.g., a subunit on the back of the cell) is moved, this procedure corresponds to a shift of the cytosol through the whole cell. Note that in the present model all subunits carry the same properties. It is only when the subunits store other properties, such as for example integrin expression, that an explicit copy algorithm of properties has to be used. When the movement of a border subunit would cause the subunits of the cell to become disconnected the movement is suppressed. For example, a subunit in the middle of a tail with a width of one subunit only cannot be moved.

Note that the border subunits are not necessarily moved to the target point nearest to $\vec{b}_{\text {virtual }}$ because this would lead to movements reflecting the lattice symmetry. Instead, we allow for deviations from the nearest target point and in this way reduce anisotropic effects on the cell movement. Within the set of free neighbor points of the cell, i.e., possible target points, only those points are considered which are within a distance $d_{i}$ of the virtual barycenter, where

$$
d_{i} \leqslant \theta\left(d_{\text {far }}-d_{\text {near }}\right)+d_{\text {near }},
$$



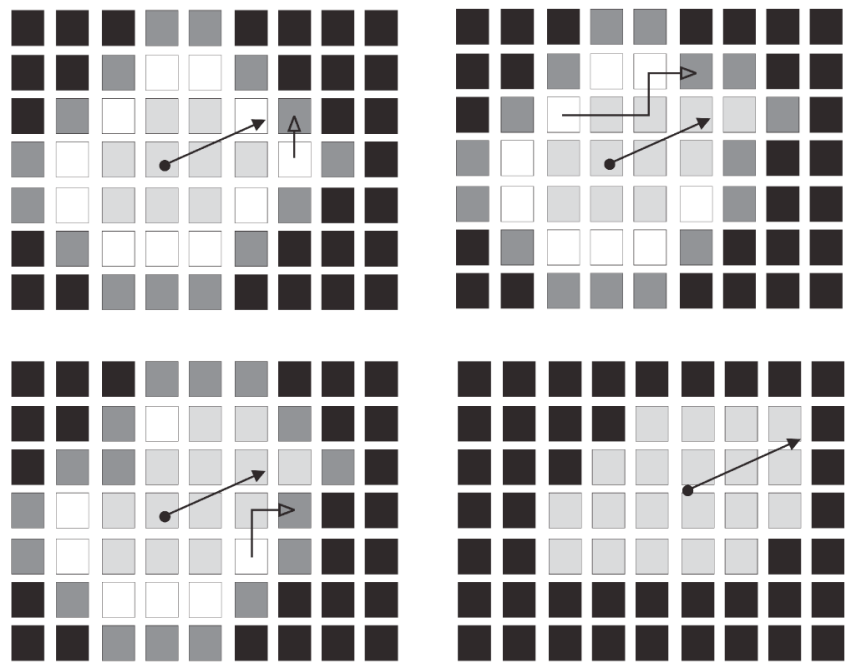

FIG. 1. A schematic description of cell movement by subunit rearrangement around a virtual barycenter. Immobile cell subunits are shown in light grey, border subunits that may be moved in white, putative free target points in dark grey, and other points in black. The current barycenter is denoted by the dot, and the cell polarity by the arrow attached to it. The arrow points the position of the virtual barycenter at the border of the cell. The starting position is shown on the top left. A random white subunit is chosen and moved towards the target point nearest to the virtual barycenter (open arrow upper panel). The result is shown in the lower panel. Note that cell subunits that are neighbors of the target point are removed from the to move list (turning them from white to light grey). In the upper right panel another random (white) subunit is moved towards the target point nearest to the virtual barycenter (open arrow). In that case the moved subunit is at the back of the cell and all cytosol subunits in between are shifted correspondingly. The result is shown in the lower left panel. The procedure is repeated (the open arrow in the lower left panel shows a possible next step) until all white subunits have been moved or removed from the to move list. A possible final constellation with a recalculated barycenter is shown in the lower right panel.

$$
\theta=\frac{\exp \left(-\frac{d_{\text {far }}-d_{\text {near }}}{\alpha d_{\text {near }}}\right)+\beta}{1+\beta \exp \left(-\frac{d_{\text {far }}-d_{\text {near }}}{\alpha d_{\text {near }}}\right)},
$$

and $d_{\text {far }}$ and $d_{\text {near }}$ are, respectively, the distance of furthest and nearest point to the virtual barycenter within the set of free neighbor points of the cell. The target point is chosen randomly within this reduced set of points. In the limit of $\alpha \rightarrow 0$ and $\beta=0$ only the target point nearest to the virtual barycenter is considered. Large values of $\alpha$ and $\beta$ reduce anisotropy but also limit cell elongation. We chose the smallest values such that anisotropic effects are not detectable by eye, leading to $\alpha=\beta=0.2$. However, the model behavior is robust against changes of these values as long as both values remain in the range between 0.1 and 0.4 .

The tolerated deviations from the target point nearest to the virtual barycenter as described by Eq. (2) are designed to be adaptive to the deformation state of the cell. For large cell deformations (i.e., $d_{\text {far }} \gg d_{\text {near }}$ ) $\theta \rightarrow \beta$ holds, while $\theta=1$ for an ideal spherical cell (i.e., $d_{\text {far }}=d_{\text {near }}$ ). However, the exact choice of Eq. (2) is arbitrary. Alternative descriptions with similar general properties are not distinguishable within the accuracy of the model.

The displacement of subunits is stopped when either no border subunit remains to be moved or the barycenter of the cell has been displaced by one lattice constant. In the new state the cell has reorganized its membrane and thus changed its shape. Thereby the total volume (the cell area in two dimensions) of the cell is conserved. Thus the movement of the cell barycenter is realized by subunit rearrangements, and inherently couples the cell movement to its deformation.

The velocity state of the cell determines the rate with which the whole procedure is started, which translates into a movement probability

$$
p_{\text {active }}=\frac{1}{\eta} \frac{\Delta t}{\Delta x} \bar{v}_{\text {active }}
$$

with $\Delta t$ and $\Delta x$ being the time and space resolution, respectively. Note that instead of moving all subunits within a single time step their movement may be distributed on $\eta$ $\geqslant 1$ time steps. Then only a fraction $1 / \eta$ of the subunits is moved per time step. This parameter (beside the lattice constant $\Delta x$ ) changes the stochastic variability of the cell movement.

It is worth mentioning that the algorithm for rearrangement of the cell subunits does not belong to the class of Markov processes if $N>1$ : Within every time step the subunits of a cell which have already moved determine which subunits may still be moved. However, all other processes are Markov-like. For example, the change of orientation depends neither on the time passed since the last change nor on the present or previous orientation.

Within this model framework every cell optionally can embed different velocity states that are characterized by different mean active velocities $\bar{v}_{i}$. They are adopted randomly and the velocity state is switched with a probability corresponding to a persistence time of velocity states $\Delta t_{v_{i}}$. The latter can be constant for all states or may be shorter for states with higher velocities. The assumption of more than one velocity state for the active movement of cells will be further discussed and compared to two-photon imaging data in Sec. III.

\section{Cell reshaping}

The second ingredient of the cell motility model concerns the cell shape stability [7]. During the procedure of active cell movement all forces that reshape the cell towards a sphere, i.e., hydrostatic pressure, reduced actin filament assembly, actomyosin contraction, or membrane surface tension, are ignored. All these forces are included in a single reshaping force. This overall elastic force drives the subunits of an elongated cell back to the current barycenter and promotes a spherical shape. Note that we do not attempt to describe the intracellular processes that underlie this total rearrangement in detail. The concentration of all these mechanisms into a single force on the cell subunits has to be considered as a phenomenological approximation. 
In complete analogy to the active cell movement, cell reshaping is represented by a velocity $v_{\text {shape }}$ of the cell subunits, which transforms into a cell border subunit movement probability $p_{\text {shape }}$ according to Eq. (4) with $\eta=1$. Subunits that are far from the barycenter are preferentially moved, i.e., if $d_{i}$ is the distance of subunit $i$ to the barycenter, then the subunit is moved with probability

$$
p_{i}=p_{\text {shape }} \frac{d_{i} N_{\text {border }}}{\sum_{j=1}^{N_{\text {border }}} d_{j}}
$$

All $N_{\text {border }}$ border subunits are moved in random order. It is worth emphasizing that the results presented below are found also for the simpler assumption $p_{i}=p_{\text {shape }}$.

The target point of every moved subunit is the free lattice point nearest to the current barycenter. Note that in contrast to the case of active movement no deviation is tolerated [i.e., $\alpha=0.01$ and $\beta=10^{-8}$ in Eq. (3)]. The reshaping forces should vanish for a spherical cell. We assume the reshaping process to scale with the elongation of the cell, $\epsilon(t)=d_{\text {far }}(t) / r_{\text {cell }}$, as a spring. Here $d_{\text {far }}(t)$ denotes the distance of the subunit farthest from the barycenter at time $t$ and $r_{\text {cell }}$ the radius of the hypothetical spherical cell with the same volume $N$. We assume that the reshaping force saturates for long elongations, setting an upper limit to the velocity of the intracellular mechanisms that reorganize the cell shape. This is described by a Hill equation with Hill coefficient 1 :

$$
v_{\text {shape }}(t)=-\bar{v}_{\text {shape }} \frac{\epsilon(t)-1}{\epsilon(t)+K_{\epsilon}-2},
$$

with $\bar{v}_{\text {shape }}$ the asymptotic subunit velocity and $K_{\epsilon}$ the elongation that corresponds to half of the maximum reshaping force. The direction of reshaping forces is defined by the difference vector between the subunit under consideration and the barycenter. Equation (6) enters the simulation via the subunit movement probability calculated with Eq. (4) for $v_{\text {shape }}(t)$ and $\eta=1$.

We would like to make the additional remark that the reshaping velocity may be interpreted as corresponding to an elastic force where all forces acting on the border subunits of the cell according to Eq. (1) are summed up to $\vec{F}_{\text {elastic. The }}$ elastic force per surface area $A$ is the tensile stress $\sigma$ of the cell which can be related to the elasticity modulus $E$ :

$$
\sigma=\frac{\left|\vec{F}_{\text {elastic }}\right|}{A}=E \frac{\Delta x_{\max }-r_{\text {cell }}}{r_{\text {cell }}}=E(\epsilon-1) .
$$

If these properties are measured for the cell type under consideration, then the reshaping velocity $\bar{v}_{\text {shape }}$ may be calculated, thus providing a consistency check.

\section{Space resolution}

On the conceptual level the model is fully formulated in terms that are independent of the time or space resolution. In particular, a formulation in terms of subunit velocities instead of diffusion constants is essential in this context. Using diffusion constants to define cell motility effectively intro- duces a persistence of the random walk proportional to the lattice constant $\Delta x$. Therefore the diffusion constant would have to be interpreted relative to the lattice resolution. This ambiguity is avoided by relying on velocities. However, an effective diffusion constant can be attributed to the movement by interpreting the persistence time $\Delta t_{\text {persist }}$ of the orientation of the cell (together with the mean velocity of active movement $\left.\bar{v}_{\text {active }}\right)$ as lattice constant $\Delta x_{\text {persist }}$ of a virtual lattice, in which the orientation is changed in every time step:

$$
\begin{aligned}
D_{\text {persist }} & \equiv p_{\text {persist }} \frac{\Delta x_{\text {persist }}^{2}}{2 d \Delta t_{\text {persist }}} \\
& =\bar{v}_{\text {active }} \frac{\Delta t_{\text {persist }} \frac{\Delta x_{\text {persist }}^{2}}{\Delta x_{\text {persist }}}=\bar{v}_{\text {active }}^{2} \frac{\Delta t_{\text {persist }}}{2 d},}{2 d t_{\text {persist }}}
\end{aligned}
$$

with $d$ the dimension of the lattice.

The stochastic diversity of the results nevertheless depends on the spatial resolution for small numbers of subunits. Within that diversity we can test that the results are independent of the lattice constant $\Delta x$ by performing in silico experiments in a regime where the stochastic width is primarily due to the probabilistic nature of the model and not due to small numbers of subunits.

\section{RESULTS}

The application of this model to specific cell types involves a small number of parameters only. Given the size of the cells the lattice constant $\Delta x$ determines the number of cell subunits $N$ that represent the cell in the model. The cell properties to be determined for every cell type include (i) the number of velocity states for active movement and the corresponding mean velocities $\bar{v}_{\text {active }}$, (ii) the persistence time $\Delta t_{\text {persist }}$ for every active velocity state, (iii) the reshaping velocity $\bar{v}_{\text {shape }}$, and (iv) the elongation corresponding to the half maximum reshaping force $K_{\epsilon}$. The artificial parameter $\eta$ that distributes the active cell movement on different time steps is used to adapt the stochastic variability of the model behavior. The natural choice is $\eta=1$, i.e., all subunits are moved within the same time step. The parameters $\alpha$ and $\beta$, which reduce anisotropic effects, are considered to be model intrinsic and will be kept constant. There are no further parameters in the model.

\section{A. A purely stochastic interpretation of the data}

In the following we see if the in vivo measurement of TC and $\mathrm{BC}$ motility in lymph nodes [1] can be repeated in silico using our model. The resolution of the lattice is set to $\Delta x$ $=0.4 \mu \mathrm{m}$. This implies TC and BC to consist of $N=241 \mathrm{sub}-$ units. As for the in vivo case, cell velocities are measured at intervals of 10 seconds in silico (note that the time interval is relevant and changes the general properties of the measurement [12]). Time courses have been registered for $12 \mathrm{~min}-$ utes. A total of 3277 measurements for TC and 3251 for BC have been carried out in vivo [1]. Thus the number of cells to be observed is determined to be 46 . The mean velocity in silico is fixed to the mean value measured in vivo of $\bar{v}_{\text {active }}^{\mathrm{TC}}$ $=10.8 \mu \mathrm{m} / \mathrm{min}$ for $\mathrm{TC}$ and to $\bar{v}_{\text {active }}^{\mathrm{BC}}=5.1 \mu \mathrm{m} / \mathrm{min}$, slightly 

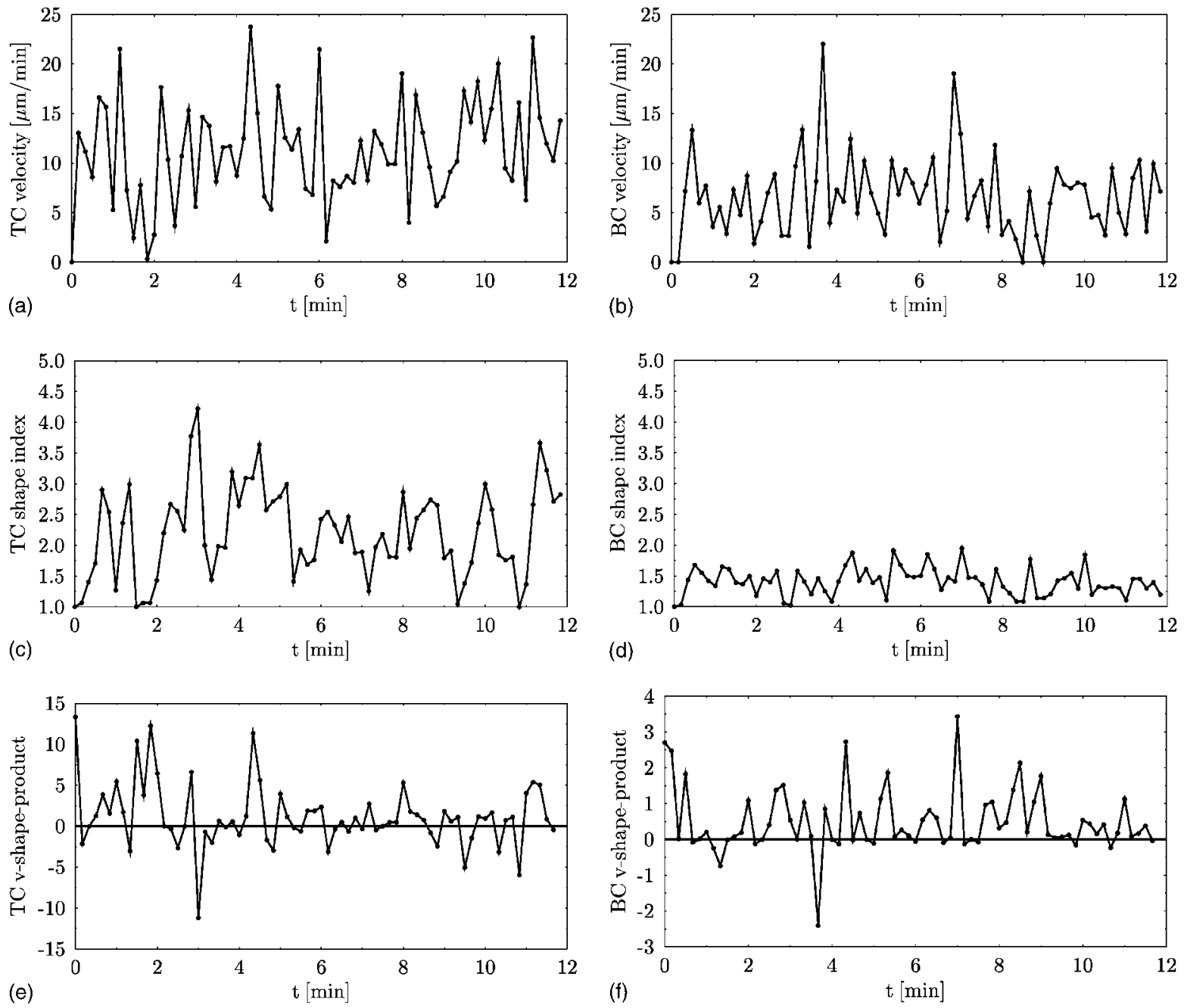

FIG. 2. The in silico time courses of velocity, shape index, and their product [defined in Eq. (9)] for TC (left panels) and BC (right panels). A single active velocity state is assumed $(10.8 \mu \mathrm{m} / \mathrm{min}$ for $\mathrm{TC}$ and $5.1 \mu \mathrm{m} / \mathrm{min}$ for BC). The spatial resolution is $0.4 \mu \mathrm{m}$.

below the measured mean value for BC (see below for explanation). The orientation persistence of the cells has been found in vivo to be between 1 and 3 minutes. Thus the value of $\Delta t_{\text {persist }}=2$ minutes is assumed in silico. The reshaping force, and the elongation corresponding to the half maximum reshaping force are chosen such that the shape index is in the correct range. The shape index is defined as the ratio of the axis length in direction of cell polarity to the axis length perpendicular to it. Note that the direction of cell polarity does not necessarily coincide with the long axis. However, it turns out that this is a good approximation to the ratio of the real longest to shortest axis. This infers $\bar{v}_{\text {shape }}^{\mathrm{TC}}=2 \mu \mathrm{m} / \mathrm{min}$ and $K_{\epsilon}^{\mathrm{TC}}=2.5$ for $\mathrm{TC}$, while for $\mathrm{BC}, \bar{v}_{\text {shape }}^{\mathrm{BC}}=4 \mu \mathrm{m} / \mathrm{min}$ and $K_{\epsilon}^{\mathrm{BC}}=1.3$.

\section{Velocity time course}

The time courses of single representative TC and $\mathrm{BC}$ as found by the model are shown in Fig. 2. The time course shows maximum velocity around $25 \mu \mathrm{m} / \mathrm{min}$ for TC and around $14 \mu \mathrm{m} / \mathrm{min}$ for $\mathrm{BC}$ (with single higher peak velocities), as found in vivo (see [1], Fig. 2C and D). TC and BC are rarely found at rest. In silico this is related to random movements of some subunits around the optimal shape even when little or no active movement occurs. This always induces small displacements of the barycenter.

The stochastic nature of the in silico experiment is also the origin of the changes between fast and slow active movements of the cells. Thus the diversity of observed cell velocities is a pure result of stochasticity and not of active changes of cell states.

\section{Time course of shape index}

The time courses of the shape index (defined as ratio of long to short axis) are shown for TC and BC in Fig. 2 (central panels). TC show strong alterations between elongated and spherical (shape index of 1) states. The shape index of $\mathrm{BC}$ shows fewer alterations and is bounded by the value of 2 .

The range of the shape index is determined by both the assumed mean velocity of the cells and the reshaping forces. The mean velocities are determined by the velocity distribution found by two-photon imaging. The difference in mean velocity between $\mathrm{TC}$ and $\mathrm{BC}$ reduces $\mathrm{BC}$ elongations considerably. Thus the lymphocyte motility already determines, to a large degree, cell shape stability. However, in vivo two- 
photon data still show less elongation for $\mathrm{BC}$. The larger reshaping forces in $\mathrm{BC}$ that have been assumed here account for that difference.

The width of the shape index alterations are-as for the velocity time course-a result of stochasticity only.

\section{Correlation of velocity and shape index}

The correlation of cell velocity and cell elongation is intrinsic to the model as cell migration is realized by the movement of its subunits. We calculate this correlation $\bar{c}$ for single time courses according to

$$
\bar{c}=\sum_{i=1}^{3266} \frac{\left(\delta\left(t_{i}\right)-\bar{\delta}\right)\left(v\left(t_{i}\right)-\bar{v}\right)}{\left[\sum_{j=1}^{3266}\left(\delta\left(t_{j}\right)-\bar{\delta}\right)^{2} \sum_{k=1}^{3266}\left(v\left(t_{k}\right)-\bar{v}\right)^{2}\right]^{1 / 2}},
$$

where $\bar{\delta}$ and $\bar{v}$ denote the mean values of the shape index and velocity, respectively. The correlation fulfills the condition $-1 \leqslant \bar{c} \leqslant 1$. Values near 1 or -1 correspond to strong positive or negative correlation of velocity and elongation, respectively, while values near 0 are adopted in case of uncorrelation. The time course of the product in the numerator is shown in Fig. 2 (lower panels). This value is not the correlation itself but a measure of the contribution of a specific cell to the correlation at every time point. The product is dominantly positive and reflects the coupling of cell motility to cell elongation. Interestingly, negative products in most cases occur after changes of orientation of the cells. The cells have to repolarize after changes of orientation such that the directions of movement and of elongation are uncorrelated for a short period. This is even more pronounced the more the cells are elongated. Indeed, the correlation is $\bar{c}=30 \%$ for $\mathrm{TC}$ and $54 \%$ for $\mathrm{BC}$ in the case shown in Fig. 2. More generally the average over 46 simulations is $\bar{c}=32 \% \pm 9 \%$ for TC and $58 \% \pm 9 \%$ for BC. Thus, the reorientation phases reduce the correlation found for rather elongated TC compared to more spherical $\mathrm{BC}$.

\section{Velocity distribution}

The velocities measured in silico in the time courses of 46 $\mathrm{TC}$ and $\mathrm{BC}$ are summed up and presented in a histogram as average counts per cell including one standard deviation (see Fig. 3(a),(b)). The in vivo results are reproduced for comparison (see black bars). According to the assumption of a single velocity state we find smooth velocity distributions for both cell types. The width of the TC distribution is larger compared to that for $\mathrm{BC}$ and corresponds well to the width found in vivo for both TC and BC. Note that the width has not been fitted. We used $\eta=1$, i.e., all subunits of a cell are moved within the same time step for both cell types. Thus, the resulting width is a prediction of the model and can be interpreted as the natural stochastic width that results from the values used for cell velocity and reshaping forces.

The maximum of the velocity distribution (averaged per cell) is larger for BC than for TC. A higher maximum value appears to result in a smaller width of the velocity distribution for $\mathrm{BC}$. The values can be compared quantitatively to
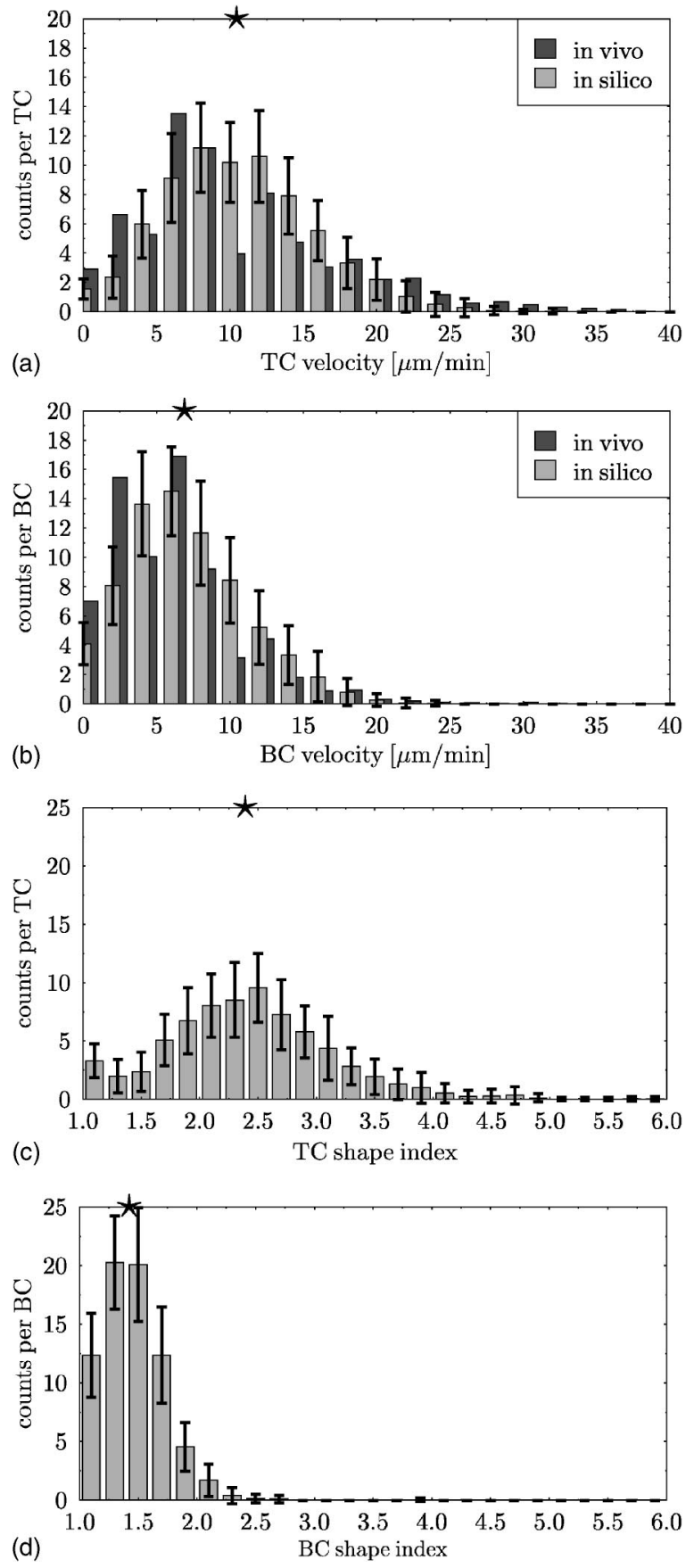

FIG. 3. For the in silico experiment in Fig. 2, the velocity and shape index distributions are averaged over all 46 considered TC [panels (a) and (c)] and BC [panels (b) and (d)]. The error bars correspond to one standard deviation and the stars to the total mean values observed in silico. The dark grey bars show the in vivo results ([1], Fig. 2C and D; data kindly provided by Mark Miller and Michael Cahalan).

the total sum of all in vivo observed velocities (see [1], Fig. $2 \mathrm{C}$ and $\mathrm{D}$ ) by multiplying the average counts with the total number (46) of considered cells. At the maximum of the velocity distribution this leads to $515 \pm 138$ counts for TC and $667 \pm 138$ for BC. The in vivo values of 622 and 778 , respectively, lie within one standard deviation. 
The mean velocity of TC is found to be $10.4 \mu \mathrm{m} / \mathrm{min}$ and corresponds to the value assumed for active movement of $10.8 \mu \mathrm{m} / \mathrm{min}$. This is in no way self-evident and depends on the relation of cell movement induced by reshaping forces and active movement. The assumed value determines only the latter. Thus we can conclude that only a minor part of TC movement is due to reshaping forces acting on deformed cells. For BC the mean velocity is found to be $6.9 \mu \mathrm{m} / \mathrm{min}$ which compares with $6.4 \mu \mathrm{m} / \mathrm{min}$ in vivo. This value is larger than the value assumed for active movement of $5.1 \mu \mathrm{m} / \mathrm{min}$. This points to a larger fraction of $\mathrm{BC}$ movement as a result of cell reshaping forces.

For a more quantitative comparison with the experimental data we calculate a deviation factor

$$
\sigma_{N}^{2}=\frac{1}{N} \sum_{i=1}^{N} \frac{n\left(v_{i}\right)-n\left(v_{i}^{\exp }\right)}{n\left(v_{i}^{\exp }\right)},
$$

where $n\left(v_{i}\right)$ and $n\left(v_{i}^{\exp }\right)$ are the counts at velocity $v_{i}$ in silico and in vivo, respectively, and $N$ is the total number of counts. This leads to $\sigma_{25}^{2}(T C)=0.54$ and $\sigma_{23}^{2}(B C)=0.38$ for the $\mathrm{T}$ cells and B cells in Fig. 3, respectively. For both cell types the deviation factor is dominated by the velocity counts at $10 \mu \mathrm{m} / \mathrm{min}$. Simulations with more than a single velocity state of the cells will be compared with these values.

\section{Shape index distribution}

In analogy to the velocity distribution, a shape index distribution is evaluated on the basis of $46 \mathrm{TC}$ and BC (see Fig. $3(\mathrm{c}),(\mathrm{d}))$. The shape index distribution is similar to the velocity distribution. However, due to the larger reshaping forces in $\mathrm{BC}$ the model predicts a shift of the $\mathrm{BC}$ distribution to smaller elongations, and substantially larger peak elongation for $\mathrm{TC}$ than for $\mathrm{BC}$. The average shape indices are 2.4 and 1.4 for $\mathrm{TC}$ and $\mathrm{BC}$, respectively.

\section{Cell tracking and territory explored}

The tracks of all cells are followed and superimposed on a single figure (see Fig. 4). The territory explored by the TC and $\mathrm{BC}$ in silico is consistent with the one found in vivo (see superimposed tracks of all cells in [1], Fig. 3A and B). Note that single TC reach a distance of about $100 \mu \mathrm{m}$ from their original position while most $\mathrm{BC}$ do not reach a distance of $50 \mu \mathrm{m}$ from their original position.

\section{The mean displacement of lymphocytes}

The displacement of TC and BC from their starting position is followed with time. The mean displacement of the 46 cells is plotted against the square root of time including one standard deviation (see Fig. 5). For both cells we find approximately a straight line, agreeing with in vivo results. The mean displacement in silico after $t=12$ minutes (i.e., $t^{1 / 2}$ $=3.5$ ) of $x=56 \mu \mathrm{m}$ for TC and $x=25 \mu \mathrm{m}$ for BC is in excellent agreement with the values found in vivo (see dotted line in Fig. 5). From these values we can deduce an underlying diffusion coefficient $D_{\text {observed }}^{\mathrm{TC} / \mathrm{BC}}=x^{2} /(2 d t)$ which gives
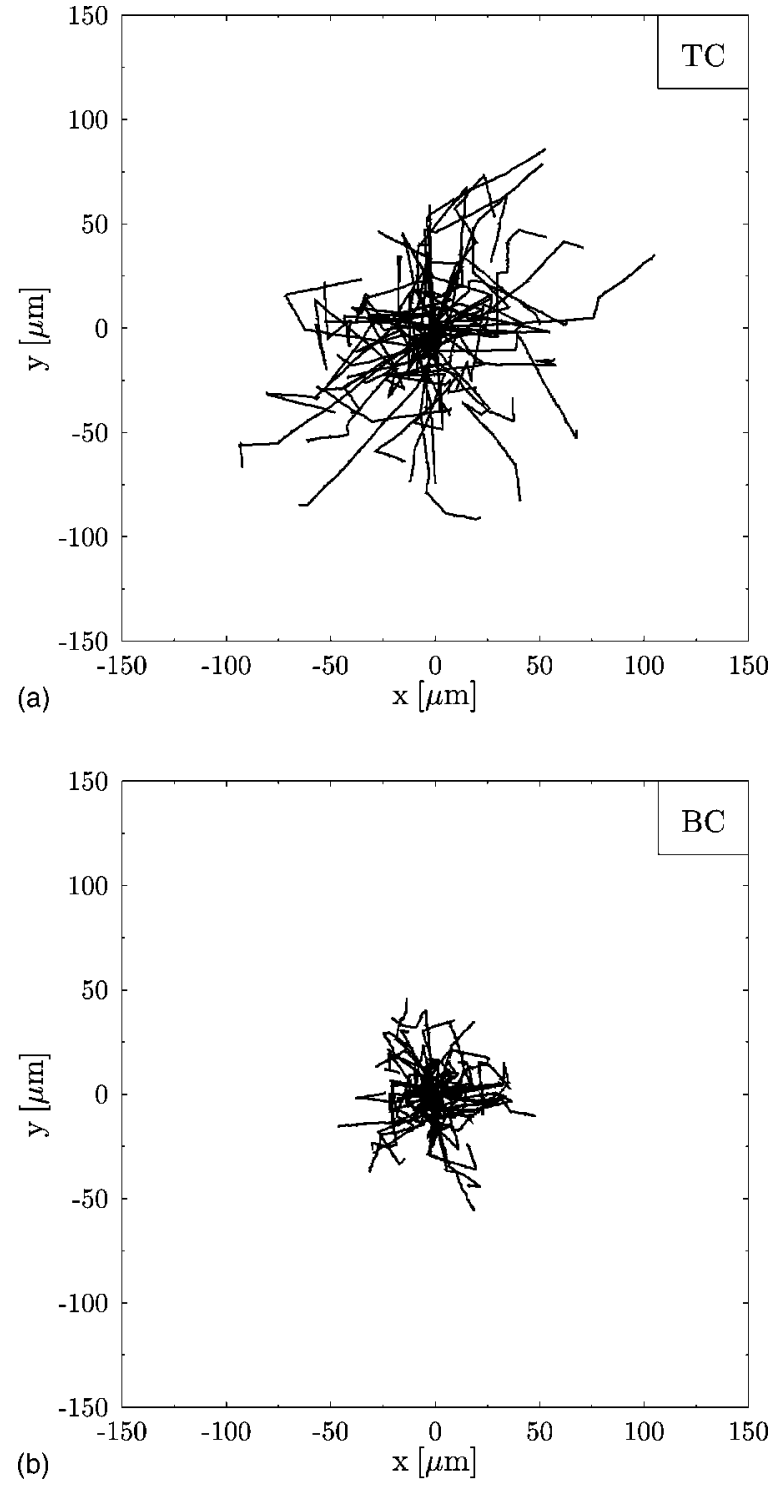

FIG. 4. Superimposed tracks of 46 TC (upper panel) and BC (lower panel). Data from the same in silico experiment as in Fig. 2.

$$
D_{\text {observed }}^{\mathrm{TC}}=65 \frac{\mu \mathrm{m}^{2}}{\min }, \quad D_{\text {observed }}^{\mathrm{BC}}=13 \frac{\mu \mathrm{m}^{2}}{\mathrm{~min}} .
$$

Note that according to Eq. (8) this corresponds to persistence times of orientation $\Delta t_{\text {persist }}$ of 2.2 and 2.0 minutes for TC and $\mathrm{BC}$, respectively, i.e., the value assumed in the simulation. This supports the consistency of the model results.

The lag time before the onset of the linear behavior in Fig. 5 appears to be longer in silico than in vivo. In silico the lag time is identical to the assumed persistence time, as it should be. The in vivo data show shorter directional memory but the same persistence times are observed. On longer time scales in vivo displacements are slightly smaller than expected from the straight line. Therefore it is likely that the rather short lag time in vivo is an artifact.

\section{Concluding remarks}

The adjustment of reshaping and active movement velocities are sufficient within our model to reproduce the main TC 

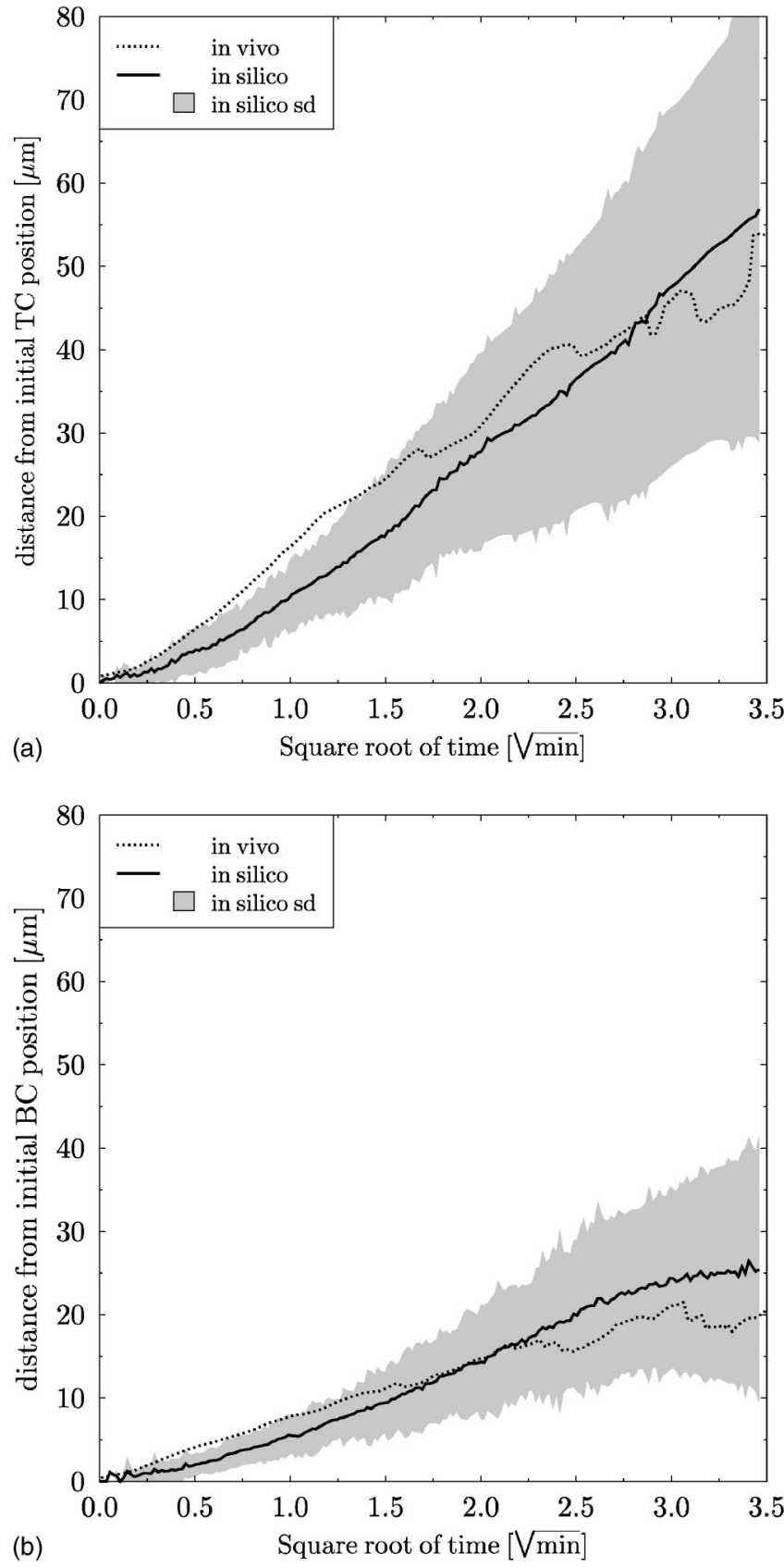

FIG. 5. The progress of displacement from the initial position averaged over TC (upper panel) and BC (lower panel). The shaded area corresponds to one standard deviation (sd) in silico. The data stem from the same in silico experiment as in Fig. 2 and are compared to the in vivo results (dotted line, data from [1], Fig. 3D, kindly provided by Mark Miller and Michael Cahalan).

and $\mathrm{BC}$ properties as found with two-photon measurement. No further assumptions are needed. An important conclusion of the in silico experiment is that no switch of the internal state of the cell is necessary in order to produce the alterations in the single cell velocity time course as well as in the shape index time course. Thus the model-intrinsic stochastic width of the cell movement is sufficient in order to generate the diversity of velocities observed experimentally. This result is underlined by the fact that the stochastic diversity of
TC and BC differ in vivo, which turns out to be reproduced in silico without tuning the stochastic width.

Note, however, that in vivo a velocity near the mean velocity remains underpopulated for TC and BC (see Fig. 3, black bars), while in silico the velocity distribution is monotonic on both sides of the maximum. For BC the in vivo value lies within the statistical width, whereas for TC it is included within 3 standard deviations of the in silico experiment and, therefore, this has to be considered as a disagreement between in vivo and in silico results. This calls into question the in silico assumption of a single velocity state. Instead one may think of a superposition of more distinct velocity states that are adopted by the cell according to some unknown environmental change, or simply randomly. This will be further elaborated below.

\section{B. Changing spatial resolution}

The same in silico experiments are repeated with half spatial resolution of $a=0.8 \mu \mathrm{m}$, i.e., cells with 60 subunits. The results for BC are shown in Fig. 6. Here the same parameter set $\left(\bar{v}_{\text {shape }}=4 \mu \mathrm{m} / \mathrm{min}, K_{\epsilon}=1.3\right.$, and $\left.\bar{v}_{\text {active }}=5.1 \mu \mathrm{m} / \mathrm{min}\right)$ as for higher resolution $(\Delta x=0.4 \mu \mathrm{m})$ has been used. The resulting characteristic data are similar to the high resolution case: mean velocity of $7.0 \mu \mathrm{m} / \mathrm{min}$, mean shape index of 1.6 , summed velocity histogram with $722 \pm 175$ counts at the maximum, and mean displacement after 12 minutes of $23 \mu \mathrm{m}$. The deviation factor according to Eq. (10) is $\sigma_{23}^{2}$ $=0.43$.

This result confirms that the model has been formulated in terms independent of the lattice resolution. However, a major precondition of this result is that the parameters are chosen in such a way that the stochasticity of the result is mainly due to the probabilistic nature of the model and not due to stochastic noise because of small subunit numbers. In the case of $\mathrm{BC}$ this is ensured by a relatively large reshaping force. In contrast the reshaping force of $\mathrm{TC}$ is relatively small. Therefore, a corresponding experiment with lower resolution for TC requires the parameter $K_{\epsilon}$ to be changed from 2.5 to 1.3 to obtain the same result.

For higher resolutions the results remain independent of the resolution because the stochastic noise due to small subunit numbers becomes negligible (data not shown).

\section{C. $T$ cells with two velocity states}

In order to try to capture the in vivo behavior which is suggestive of two velocity peaks, we introduce two velocities for active movement $\left(\bar{v}_{\text {active }}=16,8 \mu \mathrm{m} / \mathrm{min}\right)$. The width of the velocity distribution has to be considerably reduced. The model's intrinsic stochasticity can be changed by distributing every cell movement on several $(\eta=100)$ time steps. The change of velocity states is correlated with the change of polarity, thus coupling cell velocity and reorientation (see Fig. 7, upper panel). A less populated mean velocity is observed.

If both processes, reorientation and change of velocity state, are decoupled from each other, a similar result is found (see Fig. 7 lower panel). Obviously, it is not necessary to couple the dynamics of orientation and velocity state in order 

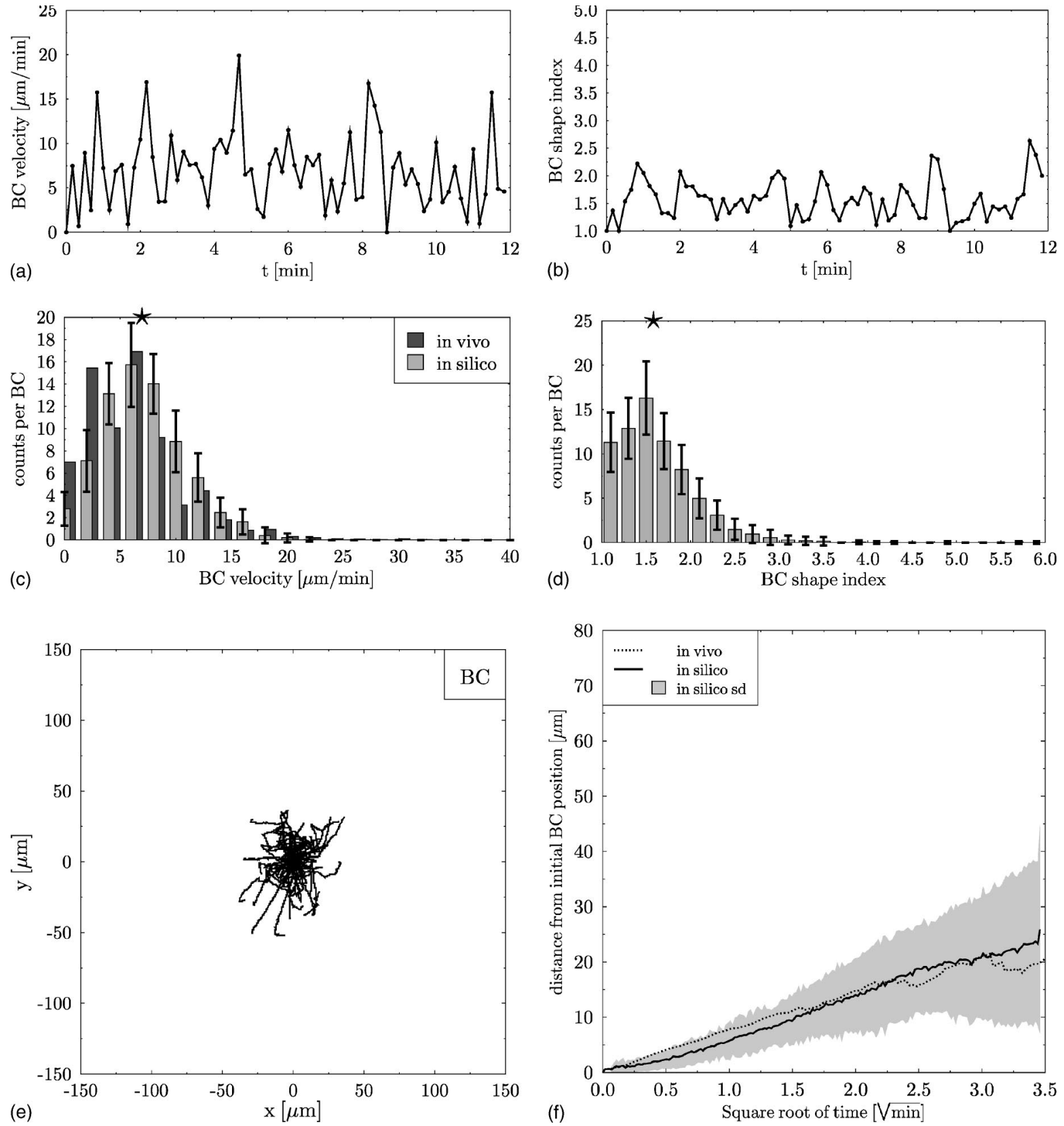

FIG. 6. The BC behavior with lower resolution of $0.8 \mu \mathrm{m}$ and the same parameter set as in Figs. 2-5. In vivo data kindly provided by Mark Miller and Michael Cahalan.

to find a depopulated mean velocity in the velocity distribution as experimentally observed.

The total width of the velocity distribution is incorrect for very high and very low velocities which, in contrast to the in vivo results, are not sufficiently populated in the in silico experiment. This is affirmed by the deviation factor $\sigma_{25}^{2}$ $=0.75$ and 0.74 for both simulations in Fig. 7 , respectively. The contribution from the critical $10 \mu \mathrm{m} / \mathrm{min}$ count is reduced by half. However, the errors related to high velocities are considerably increased. This is a result of the small stochastic width that had to be assumed in order to explain the depopulated mean velocity in the in vivo velocity distribution. Thus the depopulated mean velocity and the counts of very high and low velocities as observed in vivo rule out the hypothesis that TC switch between two velocity states.

\section{D. $T$ cells with three velocity states}

A similar in silico experiment can be done with three velocity states. The assumption of equal persistence times for all three states leads to more pronounced high velocities in contradiction to the two-photon data. However, reducing the persistence time of the highest velocity state by a factor of 2 leads to a more reasonable scenario (see Fig. 8). The deviation factor [see Eq. (1) $] \sigma_{25}^{2}=0.52$ is of similar quality as compared to the single velocity state scenario. The contributions to this factor are smoothly distributed over the whole velocity range. Similar results are found when both high ve- 

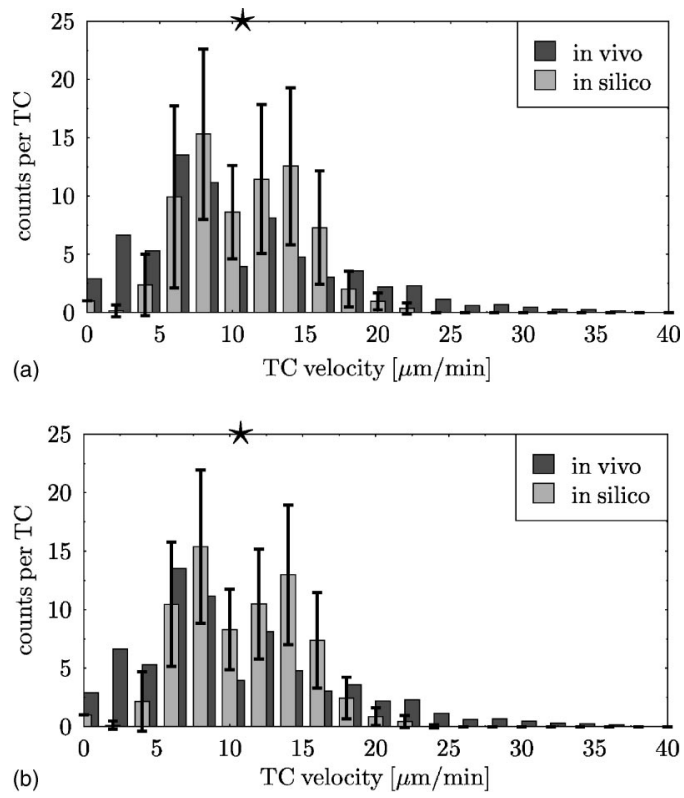

FIG. 7. The velocity distribution of TC in an in silico experiment assuming two active velocity states of 16 and $8 \mu \mathrm{m} / \mathrm{min}$. The switch of velocity states is correlated with the change of orientation in the upper panel, but not correlated in the lower panel. In vivo data kindly provided by Mark Miller and Michael Cahalan.

locity states are assumed to have shorter persistence compared to the low velocity state. Note that the depopulated average velocity is not reflected in the shape index. The latter
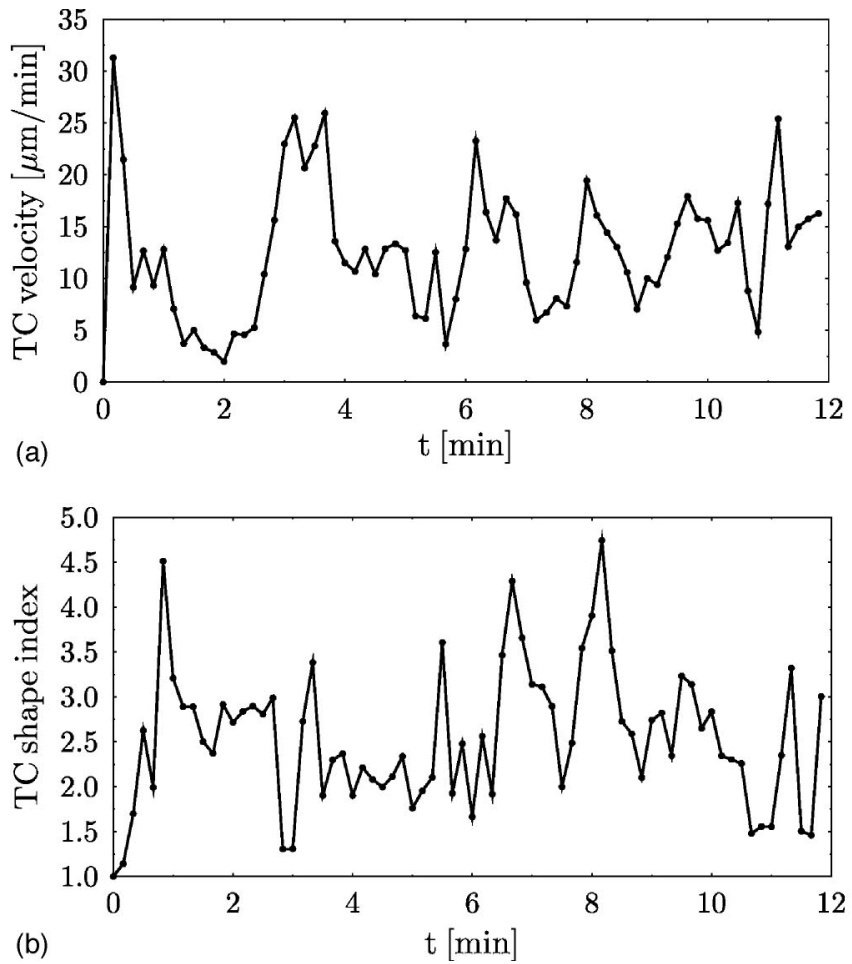

displays a rather large width that is induced by three different velocity states, each corresponding to a different average elongation.

The persistence of velocity states has to be reduced to 0.5 minutes in order to obtain the same frequency of velocity changes as in vivo. Representative results with persistence times that are too long are shown in Fig. 9. Note the small stochastic variations around the mean velocity attributed to the various velocity states of the cell. If only one velocity state is assumed the correct frequency of velocity changes is based on the stochastic width of the cell velocities only. In the case of more velocity states, the stochastic width is reduced and the velocity changes are to be interpreted as active changes of the velocity with some frequency.

An analogous analysis holds true for $\mathrm{BC}$, which is not shown here as it does not add new insights.

\section{CONCLUSIONS}

We have presented the new model hyphasma for cell motility and shaping. Cell shape and movement are reduced to the dynamics of the cell subunits that represent the cell membrane. The dynamics are described by two contributions: (i) Rearrangement of subunits with respect to a virtually shifted barycenter of the cell (active movement) and (ii) rearrangement of the subunits with respect to the actual barycenter (reshaping forces). The subunit movement is realized according to heuristic rules that are interpretable as physical quantities.
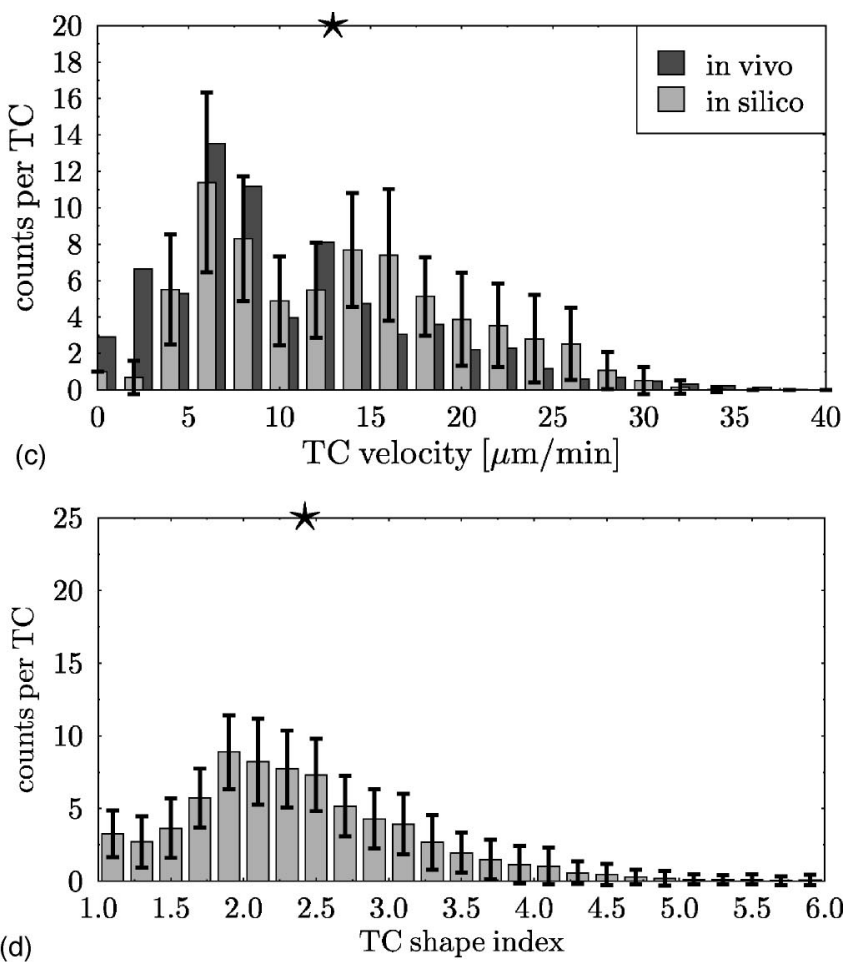

FIG. 8. TC in an silico experiment with three active velocity states $(24,15$, and $6 \mu \mathrm{m} / \mathrm{min})$. The switches between velocity states are uncorrelated with orientation changes. The persistence of the velocity states is 0.5 min for both low velocity states and 0.25 min for the highest velocity state. The right panels show the distributions per cell averaged over 46 TC. In vivo data kindly provided by Mark Miller and Michael Cahalan. 
The comparison of the model results with experiment revealed that these two ingredients, i.e., active movement and reshaping forces, are sufficient in order to describe lymphocyte motility data as found by two-photon imaging. Assuming a single velocity state of lymphocytes describes all the data for TC and BC. Thus the model confirms that the movement of lymphocytes in secondary lymphoid organs can be described as a random walk with persistence of orientation of 2 minutes.

The dynamics of the shape index found in experiment are in large parts inferred by the difference in mean velocities between TC and BC. This reflects the model assumption that cell movements are performed by cell subunit displacements, i.e., by cell deformation. However, in order to quantitatively reproduce the data within the model the reshaping forces had to be doubled for $\mathrm{BC}$ with respect to TC. This can neither be a difference in hydrostatic pressure nor in surface tension of the membrane. The difference has to be interpreted in terms of different cytoskeleton dynamics in $\mathrm{TC}$ and $\mathrm{BC}$.

However, these results ignore a depopulated mean velocity in the velocity distribution which is observed in vivo for TC. On first sight the data suggest that lymphocytes randomly switch between two velocity states. However, assuming two velocity states in silico, the model results clearly imply a contradiction between the depopulation of the mean velocity and the total width of the distribution. This contradiction can only be resolved by assuming an asymmetric distribution of cell velocities around both mean velocities. It is difficult to imagine a mechanism that breaks the symmetry of the stochastic width, so we consider this scenario to be rather unlikely.

The depopulated mean velocity observed experimentally can, however, be explained by the assumption of three or more velocity states. The velocity cell distribution is reproduced when the highest velocity state has shorter persistence times compared to both low velocity states. More generally, the persistence time of all velocity states has to be decoupled from the persistence time of the orientation (thus implying two different mechanisms for orientation and velocity state regulation). This is clearly inferred by the variability of the time courses of velocity and shape index: The assumption of three velocity states is possible only if the stochastic width is considerably reduced. The in vivo variability of the time courses is then restored in silico by a more frequent change of velocity states.

The latter point implies different interpretations of the width of the velocity distribution: Either, the depopulated mean velocity is ignored and a single velocity state assumed. Then the variability of the velocity time courses is a result of stochastic width only. Alternatively, the depopulated mean velocity is explained by at least three velocity states of lymphocytes. Then the variability of the velocity time course is a result of an active change of the internal state of the cell (with only small stochastic perturbations).

In real secondary lymphoid tissue, lymphocytes move in a labyrinth of obstacles. We have therefore tested the effect of obstacles on the observed cell motility. The result is that the stochastic width of the velocity distribution is considerably enhanced. For reasonable densities of obstacles the velocity distribution becomes dominated by intermediate velocities.
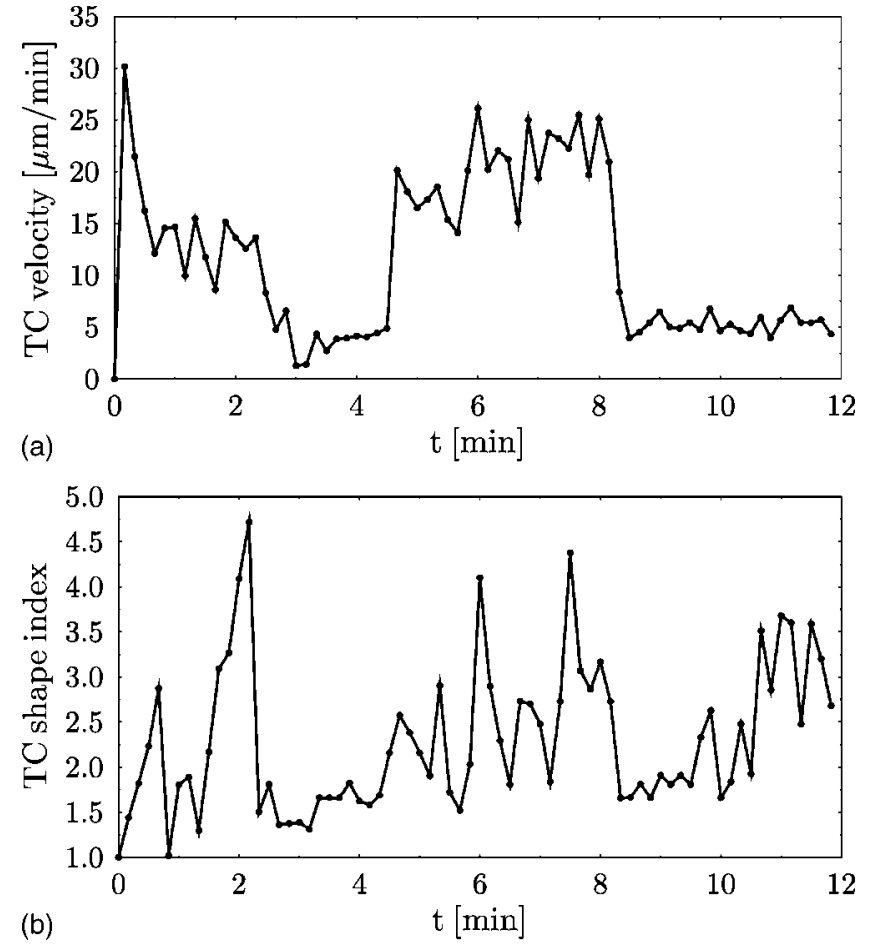

FIG. 9. TC in an silico experiment with three active velocity states of 25,15 , and $5 \mu \mathrm{m} / \mathrm{min}$ that switch uncorrelated with orientation and that persist for $2 \mathrm{~min}$. The changes of velocity state are clearly reflected in the velocity time course.

This rather intuitive result favors the scenario that TC and $\mathrm{BC}$ move in a single velocity state with large stochastic width. A depopulated mean velocity in the velocity distribution becomes rather difficult to reproduce. The purely stochastic interpretation of the velocity distribution is further supported by the observation that the width of the velocity distribution scales identically in vivo and in silico for single velocity states. In contrast, the stochastic width has to be fitted separately (using the parameter $\eta$ ) for $\mathrm{TC}$ and $\mathrm{BC}$ when a three velocity state is considered. However, it remains to be clarified in further experiments whether a depopulated mean velocity represents a real property of lymphocytes.

The present in silico experiment enforces the interpretation of lymphocyte migration as active and undirected movement with orientation persistence in the range of minutes [6]. However, we have also seen that a width of a velocity distribution may either be a result of stochastic variation or of determined active changes of internal states. This raises the question of whether the straight line found for the relation of the distance reached and the square-root of time really points to a persistent random walk. Alternatively, one may think of it to be the result of a specific and more complex chemotactic movement [13]. Indeed, not only the expression of chemokine receptors but also their pronounced expression on the leading edge of migrating cells has been observed in both TC and $\mathrm{BC}[14,15]$. The requirements for chemokine sources that lead to a seemingly random walk of lymphocytes in lymph nodes remain to be worked out in further in silico investigations. 


\section{ACKNOWLEDGMENTS}

M. M.-H. is indebted to Gernot Schaller for fruitful discussions and was supported by the Sixth EU Framework
Program. We thank Mark Miller and Michael Cahalan for providing the in vivo data that we reproduced in this article.
[1] M. J. Miller, S. H. Wei, I. Parker, and M. D. Cahalan, Science 296, 1869 (2002).

[2] P. Bousso and E. Robey, Nat. Immun. 4, 579 (2003).

[3] M. Komai-Koma, A. M. Donachie, and P. C. Wilkinson, Immunology 91, 579 (1997).

[4] C. C. Bleul, J. L. Schultze, and T. A. Springer, J. Exp. Med. 187, 753 (1998).

[5] D. J. Campbell, C. H. Kim, and E. C. Butcher, Immunol. Rev. 195, 58 (2003).

[6] S. H. Wei, I. Parker, M. J. Miller, and M. D. Cahalan, Immunol. Rev. 195, 136 (2003).

[7] W. Alt, and R. T. Tranquillo, J. Biol. Syst. 3, 905 (1995).

[8] D. Drasdo, R. Kree, and J. S. McCaskill, Phys. Rev. E 52, 6635 (1995).

[9] E. Palsson and H. G. Othmer, Proc. Natl. Acad. Sci. U.S.A.
97, 10448 (2000).

[10] G. Schaller, and M. Meyer-Hermann, Comput. Phys. Commun. 162, 9 (2004).

[11] F. Graner, and J. A. Glazier, Phys. Rev. Lett. 69, 2013 (1992).

[12] E. Méhes, A. Czirók, B. Hegedüs, T. Vicsek, and V. Jancsik, Cell Motil. Cytoskeleton 53, 203 (2002).

[13] K. M. Ansel, V. N. Ngo, P. L. Hyman, S. A. Luther, R. Forster, J. D. Sedgwick, J. L. Browning, M. Lipp, and J. G. Cyster, Nature (London) 406, 309 (2000).

[14] M. Nieto, J. M. R. Frade, D. Sancho, M. Mellado, C. MartinezA., and F. Sanchez-Madrid, J. Exp. Med. 186, 153 (1997).

[15] M. Vicente-Manzanares, M. C. Montoya, M. Mellado, J. M. R. Frade, M. A. del Pozo, M. Nieto, M. O. de Landazuri, C. Martinez-A., and F. Sanchez-Madrid, Eur. J. Immunol. 28, 2197 (1998). 